\title{
Dimensional Transmutation by Monopole Condensation in QCD
}

\author{
Y. M. Chd* \\ School of Electrical and Computer Engineering \\ Ulsan National Institute of Science and Technology, Ulsan 689-798 \\ and \\ School of Physics and Astronomy \\ Seoul National University, Seoul 151-747, Korea
}

\begin{abstract}
We compare two competing conjectures of the color confinement in QCD, the monopole condensation and the Abelian dominance, and show that it is the monopole condensation which is responsible for the confinement. To demonstrate this we present a new gauge invariant integral expression of the one-loop QCD effective action which has no infra-red divergence. With this we show that, just as the GSO-projection restores the supersymmetry and modular invariance in NSR string theory, the color reflection invariance ("the C-projection") assures the gauge invariance and the stability of the monopole condensation. This establishes the monopole dominance in QCD. In doing so we point out critical defects in the calculation of the Savvidy-Nielsen-Olesen (SNO) effective action.
\end{abstract}

PACS numbers: 12.38.-t, 12.38.Aw, 11.15.-q, 11.15.Tk

Keywords: Abelian dominance, dual Meissner effect, color reflection invariance, C-parity, monopole condensation, stability of monopole condensation, monopole dominance in QCD, color confinement

\section{INTRODUCTION}

The confinement problem in quantum chromodynamics (QCD) is probably one of the most challenging problems in theoretical physics. There have been many conjectures on the confinement mechanism, but two of them are outstanding. One is the monopole condensation, the other is the Abelian dominance [1 $[6]$. It has long been argued that the confinement in QCD can be triggered by the monopole condensation [1-3]. Indeed, if one assumes the monopole condensation, one can easily argue that the ensuing dual Meissner effect could guarantee the confinement of color. To prove the monopole condensation, however, one must first separate the monopole potential from the QCD potential gauge independently.

On the other hand the Abelian dominance proposed by 'tHooft asserts that the "Abelian part" of QCD must be responsible for the confinement [4 6]. The justification of this is that the colored (non-Abelian) gluons can not play any role in the confinement, because they (just like the quarks) describe the colored gluons which themselves have to be confined. So, only the neutral (Abelian) gluons, if at all, could possibly contribute to the confinement. In this sense, this conjecture must be true [4, 7].

The Abelian dominance has become very popular and widely been studied in lattice QCD [5, 6]. As it stands, however, it also has serious drawbacks. First of all, we must first separate the Abelian part to prove this conjecture, but it does not tell us how to do that gauge independently. The popular way to obtain the Abelian part is to choose the so-called "the maximal Abelian

*Electronic address: ymcho@unist.ac.kr gauge" [5, 6]. But strictly speaking, this is a gauge fixing. More seriously, the maximal Abelian gauge does not tell exactly what constitutes the Abelian part. Obviously the Abelian part must contain the trivial Maxwell-type Abelian potential, but this $\mathrm{U}(1)$ potential is not supposed to produce the confinement. So the Abelian part must contain something else. But the maximal Abelian gauge does not specify what is that. This means that, even if we prove the Abelian dominance, we can not tell what is really responsible for the confinement.

Fortunately we have a gauge independent Abelian projection defined by the magnetic isometry [2, 3]. An important feature of this projection is that it tells exactly what is the Abelian part. It tells that the Abelian potential is made of two parts, the non-topological Maxwell-type U(1) part and the topological non-Abelian monopole part. This is because in this projection the Abelian potential has the full non-Abelian gauge degrees of freedom, in spite of the fact that it is Abelian. So it naturally contains the topological degrees.

Furthermore, this projection separates the monopole potential gauge independently [2, 3]. This is very important because, unlike the Abelian projection based on the maximal Abelian gauge, this allows us to test not only the Abelian dominance but also the monopole dominance, and thus pinpoint exactly what is responsible for the confinement.

Implementing the gauge independent Abelian projection on lattice, the KEK lattice group led by Kondo recently have demonstrated that the confinement in QCD is due to the monopole condensation. They have shown that the confining force in SU(2) QCD comes from the Abelian part of the potential, but more precisely the monopole part of the Abelian projection [8, 9]. This clearly establishes (not just the Abelian dominance but) 


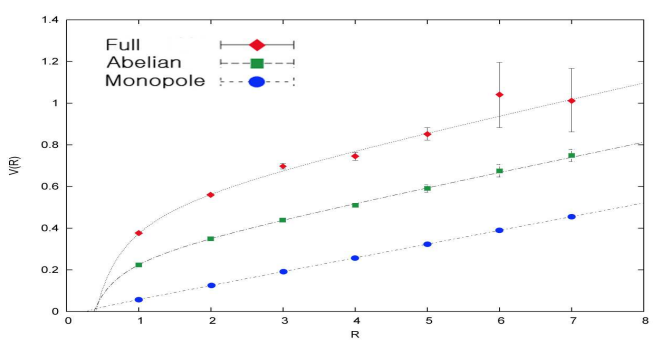

FIG. 1: The lattice QCD result which establishes the monopole dominance in SU(2) QCD. Here the confining potentials marked by red, green, and blue points are obtained with the full $\mathrm{SU}(2)$ potential, the Abelian (restricted) potential, and the monopole potential, respectively.

the monopole dominance in QCD. This remarkable result is shown in Fig 1.

Of course, the Abelian and/or monopole dominance have been studied in lattice QCD before [5, 6, 10, 11]. But these studies were based on gauge dependent separations of Abelian and/or monopole potentials, so that their results were gauge dependent. In contrast, the recent KEK calculations are explicitly gauge independent, because they are based on the gauge independent Abelian projection.

A natural way to establish the monopole condensation in QCD is to show that the quantum fluctuation triggers a phase transition similar to the dimensional transmutation observed in massless scalar QED [12]. There have been many attempts to demonstrate this. Savvidy has first calculated the effective action of SU(2) QCD in the presence of an ad hoc color magnetic background, and has almost "proved" the magnetic condensation [13].

Unfortunately, the subsequent calculation repeated by Nielsen and Olesen showed that the effective action has an extra imaginary part which destablizes the magnetic condensation [14, 15]. This is known as the instability of the "Savvidy-Nielsen-Olesen (SNO) vacuum", which has led many people to believe that it is impossible to have a stable magnetic condensation in QCD [16, 17].

The origin of this instability can be traced to the tachyonic modes in the functional determinant of the gluon loop integral. But in physics we encounter the tachyons when we do something wrong. For example, in spontaneous symmetry breaking we have tachyons when we choose the false vacuum. Similarly, in Neveu-SchwarzRamond (NSR) string we have the tachyonic vacuum when we do not make the theory supersymmetric and modular invariant with the Gliozzi-Scherk-Olive (GSO) projection [18, 19]. The question here is how to remove the tachyonic modes in the gluon functional determinant, and how to justify that.

The purpose of this paper is to calculate the one-loop QCD effective action in the presence of the monopole background, and establish the stable monopole condensation in $\mathrm{SU}(2) \mathrm{QCD}$ theoretically. Imposing the color reflection invariance (the C-parity) on the gluon functional determinant which assures the gauge invariance, we obtain a new infra-red finite integral expression of $Q C D$ effective action. From this we show that a stable monopole condensation takes place and becomes the true vacuum of $Q C D$. In particular we show that, just as the GSO projection (the G-parity) removes the tachyonic vacuum in string theory, the C-parity in $Q C D$ removes the tachyons and assures the stability of the monopole condensation. This establishes the dimensional transmutation by the monopole condensation in QCD.

It is well known that the $\mathrm{SNO}$ vacuum is not gauge invariant, so that it can not be the $\mathrm{QCD}$ vacuum even if it were stable [15]. In this paper we show another critical defect of the SNO vacuum, that it violates the parity. Since the parity is conserved in strong interaction this is a serious defect. Moreover, we show that the gauge invariant and parity conserving background is the monopole background, which is why we have chosen the monopole background in our calculation of the effective action.

In doing so we point out that the SNO vacuum is not the QCD vacuum but a vacuum of an Abelian gauge theory coupled to a massless charged vector field which has no charge conjugation invariance, which is a sick theory that has nothing to do with QCD.

The monopole condensation predicts two vacuum fluctuation modes of $\mathrm{QCD}$, the $0^{++}$and $1^{++}$magnetic glueballs which are not made of the valence gluons. This is because the monopole condensation generates two scales, the correlation length of the monopole condensation and the penetration length of the color flux.

The paper is organized as follows. In Section II we compare the 'tHooft's Abelian decomposition and the gauge invariant Abelian decomposition for later purpose. In Section III we discuss the difference between the Savvidy background and the monopole background, and show that only the monopole background is gauge invariant and parity conserving. In Section IV we review the problems of the SNO effective action. In Section V we discuss the gauge invariant calculation of QCD effective action, and show how the color reflection invariance (the C-projection) excludes the tachyonic modes from the gluon functional determinant. In particular, we obtain a new integral expression of the one-loop QCD effective action which generates the stable monopole condensation. In Section VI we discuss a more general issue, the infrared instability of the QCD effective action which originates from the absence of a mass scale, and show how the infra-red regularization by causality can restore the stability. In Section VII we emphasize that the perturbative calculation of the imaginary part can also assure the stability of the effective action. In Section VIII we derive the QCD effective potential and show that the QCD vacuum is given by the monopole condensation. This es- 
tablishes the dimensional transmutation by the monopole condensation in QCD. Finally in Section IX we discuss the physical implications of our result.

\section{ABELIAN DECOMPOSITION: A REVIEW}

To establish the Abelian dominance, we must know what is the Abelian part of QCD which is responsible for the Abelian sub-dynamics. To do that we have to make the Abelian projection. There are two competing proposals for the Abelian projection.

The popular Abelian projection proposed by 'tHooft is to remove as many non-Abelian degrees of freedom as possible by partially fixing the gauge, and to reduce QCD to an Abelian gauge theory coupled to the colored gluons [4, 5]. To explain how this proposal is supposed to work, consider the SU(2) QCD for simplicity. Let $\left(\hat{n}_{1}, \hat{n}_{2}, \hat{n}_{3}\right)$ be an arbitrary right-handed local orthonormal basis, and choose $\hat{n}=\hat{n}_{3}$ to be the Abelian (i.e., neutral) direction (which we can do without loss of generality). Now, let

$$
\begin{gathered}
\vec{A}_{\mu}=A_{\mu}^{1} \hat{n}_{1}+A_{\mu}^{2} \hat{n}_{2}+A_{\mu} \hat{n}=\mathscr{A}_{\mu}+\mathscr{X}_{\mu}, \\
\mathscr{A}_{\mu}=A_{\mu} \hat{n}, \quad \mathscr{X}_{\mu}=A_{\mu}^{1} \hat{n}_{1}+A_{\mu}^{2} \hat{n}_{2},
\end{gathered}
$$

and interprete $\mathscr{A}_{\mu}$ and $\mathscr{X}_{\mu}$ as the neutral and colored potentials, assuming the colored part transforms gauge covariantly. In this case $A_{\mu}$ has the residual U(1) gauge degrees which leaves $\hat{n}$ invariant, which makes QCD effectively an Abelian gauge theory which has the colored gluons as a source.

Here, however, we have to tell how to select the Abelian part $\mathscr{A}_{\mu}$. A popular conjecture to do that is to impose the so-called maximal Abelian gauge condition,

$$
\hat{\mathcal{D}}_{\mu} \mathscr{X}_{\mu}=0, \quad \hat{\mathcal{D}}_{\mu}=\partial_{\mu}+g \mathscr{A}_{\mu} \times
$$

The logic behind this is that $\hat{\mathcal{D}}_{\mu} \mathscr{X}_{\mu}$ becomes an isovector in color direction, so that by suppressing it with (2) one could remove as many non-Abelian degrees as possible, and thus have the Abelian (neutral) potential $\mathscr{A}_{\mu}$. This Abelian projection has been widely adopted in lattice QCD to demonstrate the Abelian dominance [5, 6].

This logic, however, has a serious defect. To fix $\mathscr{A}_{\mu}$ from (2), we must know $\mathscr{X}_{\mu}$, but obviously we need $\mathscr{A}_{\mu}$ to determine $\mathscr{X}_{\mu}$. This is a vicious circle. So it can not determine $\mathscr{A}_{\mu}$. In fact, (2) should (as we will see) more properly be interpreted as a condition on $\mathscr{X}_{\mu}$, not on $\mathscr{A}_{\mu}$. More seriously, (11) itself has the critical defect that the decomposition of the neutral and colored gluons is gauge dependent.

The other Abelian projection is to use the Abelian isometry to project the Abelian part [2, 3]. Let $\hat{n}$ be the neutral direction as before. Since this automatically determines the color directions $\hat{n}_{1}$ and $\hat{n}_{2}$, we may also call $\hat{n}$ the color direction. To make the Abelian projection we impose the maximal Abelian isometry on $\vec{A}_{\mu}$,

$$
D_{\mu} \hat{n}=\left(\partial_{\mu}+g \vec{A}_{\mu} \times\right) \hat{n}=0
$$

and obtain the restricted potential $\hat{A}_{\mu}$ which describes the Abelian sub-dynamics of QCD

$$
\begin{gathered}
\vec{A}_{\mu} \rightarrow \hat{A}_{\mu}=A_{\mu} \hat{n}-\frac{1}{g} \hat{n} \times \partial_{\mu} \hat{n}=\mathscr{A}_{\mu}+\vec{C}_{\mu} \\
\vec{C}_{\mu}=-\frac{1}{g} \hat{n} \times \partial_{\mu} \hat{n} .
\end{gathered}
$$

Notice that (3) is gauge covariant, so that this projection is gauge independent. We emphasize that (3) restricts the connection precisely to the connection which leaves the Abelian direction $\hat{n}$ invariant under the parallel transport.

Clearly $\hat{A}_{\mu}$ is different from $\mathscr{A}_{\mu}$. It includes $\mathscr{A}_{\mu}$, but also has $\vec{C}_{\mu}$ which generates a non-trivial field strength

$$
\begin{gathered}
\vec{H}_{\mu \nu}=\partial_{\mu} \vec{C}_{\nu}-\partial_{\nu} \vec{C}_{\mu}+g \vec{C}_{\mu} \times \vec{C}_{\nu}=H_{\mu \nu} \hat{n} \\
H_{\mu \nu}=-\frac{1}{g} \hat{n} \cdot\left(\partial_{\mu} \hat{n} \times \partial_{\nu} \hat{n}\right)=\partial_{\mu} \tilde{C}_{\nu}-\partial_{\nu} \tilde{C}_{\mu} \\
\tilde{C}_{\mu}=-\frac{1}{g} \hat{n}_{1} \cdot \partial_{\mu} \hat{n}_{2} .
\end{gathered}
$$

Notice that $\tilde{C}_{\mu}$ is uniquely determined up to the $\mathrm{U}(1)$ gauge transformation which leaves $\hat{n}$ invariant.

To understand the physical meaning of this, notice that $\vec{C}_{\mu}$ describes the non-Abelian monopole, because $\hat{n}$ defines the mapping $\pi_{2}\left(S^{2}\right)$ which describes the monopole topology. Indeed, when $\hat{n}=\hat{r}$ it describes precisely the Wu-Yang monopole [20, 21]. Moreover, with

$$
\hat{n}_{1}=\cos \phi \hat{\theta}-\sin \phi \hat{\phi}, \quad \hat{n}_{2}=\sin \phi \hat{\theta}+\cos \phi \hat{\phi}
$$

the corresponding $\tilde{C}_{\mu}$ becomes exactly the Dirac's monopole potential [2, 21]

$$
\tilde{C}_{\mu}=-\frac{1}{g} \hat{n}_{1} \cdot \partial_{\mu} \hat{n}_{2}=-\frac{1}{g}(1-\cos \theta) \partial_{\mu} \phi .
$$

This confirms that $\hat{A}_{\mu}$ includes the non-Abelian monopole potential, in spite of the fact that it describes the Abelian sub-dynamics.

To clarify this dual structure, notice that

$$
\begin{gathered}
\hat{F}_{\mu \nu}=\partial_{\mu} \hat{A}_{\nu}-\partial_{\nu} \hat{A}_{\mu}+g \hat{A}_{\mu} \times \hat{A}_{\nu} \\
=\left(F_{\mu \nu}+H_{\mu \nu}\right) \hat{n} \\
F_{\mu \nu}=\partial_{\mu} A_{\nu}-\partial_{\nu} A_{\mu}, \quad H_{\mu \nu}=\partial_{\mu} \tilde{C}_{\nu}-\partial_{\nu} \tilde{C}_{\mu} .
\end{gathered}
$$

So the "Abelian" field strength of the restricted potential is actually made of two field strenghts, $F_{\mu \nu}$ and $H_{\mu \nu}$, each of them given by Abelian potential $A_{\mu}$ and $\tilde{C}_{\mu}$. In 
other words the Abelian part of QCD is made of two potentials, the topological Dirac-type "magnetic" potential $\vec{C}_{\mu}$ as well as the non-topological Maxwell-type "electric" potential $\mathscr{A}_{\mu}$.

With the Abelian projection we obtain the restricted chromodynamics (RCD) which describes the Abelian sub-dynamics of QCD [2, 3]

$$
\mathcal{L}_{R C D}=-\frac{1}{4} \hat{F}_{\mu \nu}^{2}=-\frac{1}{4}\left(F_{\mu \nu}+H_{\mu \nu}\right)^{2} .
$$

As we will see RCD has the full SU(2) gauge freedom, in spite of the fact that it describes the Abelian subdynamics.

Now, we can recover the full QCD gauge potential by adding the colored part $\vec{X}_{\mu}$ to $\hat{A}_{\mu}[2,3]$

$$
\begin{gathered}
\vec{A}_{\mu}=\hat{A}_{\mu}+\vec{X}_{\mu}=A_{\mu} \hat{n}-\frac{1}{g} \hat{n} \times \partial_{\mu} \hat{n}+\vec{X}_{\mu} \\
\vec{X}_{\mu}=\frac{1}{g} \hat{n} \times D_{\mu} \hat{n}, \quad \hat{n} \cdot \vec{X}_{\mu}=0 .
\end{gathered}
$$

This is the gauge independent Abelian decomposition of the non-Abelian gauge potential, also known as ChoDuan-Ge or Cho-Faddeev-Niemi-Shabanov decomposition 22 24].

To understand the meaning of (10), consider the gauge transformation

$$
\delta \vec{A}_{\mu}=\frac{1}{g} D_{\mu} \vec{\alpha}, \quad \delta \hat{n}=-\vec{\alpha} \times \hat{n}
$$

where $\vec{\alpha}$ is an infinitesimal gauge parameter. Under this we have

$$
\begin{gathered}
\delta A_{\mu}=\frac{1}{g} \hat{n} \cdot \partial_{\mu} \vec{\alpha}, \quad \delta \hat{A}_{\mu}=\frac{1}{g} \hat{D}_{\mu} \vec{\alpha} \\
\delta \vec{X}_{\mu}=-\vec{\alpha} \times \vec{X}_{\mu} .
\end{gathered}
$$

This tells that $\hat{A}_{\mu}$ by itself describes an SU(2) connection which enjoys the full $\mathrm{SU}(2)$ gauge degrees of freedom. So it inherits the full topological properties of nonAbelian gauge theory, even though it describes only the Abelian sub-dynamics. This is why it contains the nonAbelian monopole potential, which makes RCD different from QED.

Furthermore $\vec{X}_{\mu}$ forms a gauge covariant vector field under the gauge transformation. This is because the connection space forms an affine space, so that any point of the connection space can be reached from the Abelian connection adding a gauge covariant vector field. Moreover it is colored $\left(\hat{n} \cdot \vec{X}_{\mu}=0\right)$. So we call $\hat{A}_{\mu}$ and $\vec{X}_{\mu}$ the binding and valence potential which represents the binding and colored gluons, respectively.

But what is really remarkable about (10) is that the decomposition is gauge independent. Once $\hat{n}$ is chosen, the decomposition follows automatically, regardless of the choice of gauge. In particular, the separation of the monopole potential is gauge independent [2, 3].

So using this Abelian decomposition we can perform a truly gauge independent lattice calculation to test the monopole dominance. As we have explained the result is shown in Fig. 1, where the red, green, and blue dots are obtained with $\vec{A}_{\mu}, \hat{A}_{\mu}$, and $\vec{C}_{\mu}$ [8, 9]. This clearly shows that it is the monopole which is responsible for the color confinement.

With the decomposition (10), we have

$$
\vec{F}_{\mu \nu}=\hat{F}_{\mu \nu}+\hat{D}_{\mu} \vec{X}_{\nu}-\hat{D}_{\nu} \vec{X}_{\mu}+g \vec{X}_{\mu} \times \vec{X}_{\nu}
$$

so that the Yang-Mills Lagrangian is expressed as

$$
\begin{aligned}
\mathcal{L}= & -\frac{1}{4} \vec{F}_{\mu \nu}^{2}=-\frac{1}{4} \hat{F}_{\mu \nu}^{2}-\frac{1}{4}\left(\hat{D}_{\mu} \vec{X}_{\nu}-\hat{D}_{\nu} \vec{X}_{\mu}\right)^{2} \\
& -\frac{g}{2} \hat{F}_{\mu \nu} \cdot\left(\vec{X}_{\mu} \times \vec{X}_{\nu}\right)-\frac{g^{2}}{4}\left(\vec{X}_{\mu} \times \vec{X}_{\nu}\right)^{2} .
\end{aligned}
$$

This shows that QCD can be viewed as RCD made of the binding gluons, which has the colored valence gluons as its source [2, 3].

But we emphasize that (14), strictly speaking, is different from QCD because it has more symmetry. This is because the decomposition (10) automatically enlarges the gauge symmetry 25]. Obviously it is invariant under the gauge transformation of the active type, the classical (slow) gauge transformation, described by (11) and (12). But notice that it is also invariant under the following gauge transformation of the passive type, the quantum (fast) gauge transformation,

$$
\delta \vec{A}_{\mu}=\frac{1}{g} D_{\mu} \vec{\alpha}, \quad \delta \hat{n}=0,
$$

under which one has

$$
\begin{gathered}
\delta A_{\mu}=\frac{1}{g} \hat{n} \cdot D_{\mu} \vec{\alpha}, \quad \delta \hat{A}_{\mu}=\frac{1}{g}\left(\hat{n} \cdot D_{\mu} \vec{\alpha}\right) \hat{n} \\
\delta \vec{X}_{\mu}=\frac{1}{g}\left[D_{\mu} \vec{\alpha}-\left(\hat{n} \cdot D_{\mu} \vec{\alpha}\right) \hat{n}\right]
\end{gathered}
$$

This is because, for a given $\vec{A}_{\mu}$, we can have different decompositions choosing different $\hat{n}$. Equivalently, for a fixed $\hat{n}$, we have different $\vec{A}_{\mu}$ which are gauge equivalent. So the decomposition inevitably enlarge the gauge symmetry.

The extra gauge symmetry is a generic feature of the background field formalism in quantum field theory [26, 27]. The decomposition of the field to classical and quantum parts does not tell us how to assign the symmetry of the theory to the decomposed parts, so that we have freedom to impose the symmetry to two parts. For this reason we call (14) the extended chromodynamics (ECD).

The enlarged gauge symmetry plays an important role in ECD. According to the active gauge symmetry (12), 
$\vec{X}_{\mu}$ is gauge covariant, so that the valence gluons (in principle) could acquire a mass under quantum correction. However, the passive gauge symmetry (16) makes this impossible. This is because we have to impose a gauge condition to fix the passive gauge degrees to quantize $\vec{X}_{\mu}$. A natural choice is the generalized Lorentz gauge [25]

$$
\hat{D}_{\mu} \vec{X}_{\mu}=0 .
$$

Of course we could choose other gauges (e.g., the Coulomb gauge), but the point here is that the gauge condition keeps the valence gluons massless. This assures that ECD is physically equivalent to QCD, even though it has more symmetry. This point will play an important role when we calculate the effective action of QCD with the background field method in the following.

Now, we can compare two Abelian projections (2) and (3). Clearly $\mathscr{A}_{\mu}$ and $\mathscr{X}_{\mu}$ in (2) play the role of $\hat{A}_{\mu}$ and $\vec{X}_{\mu}$ in (3), so that the maximal Abelian gauge corresponds to the Lorentz gauge (17). But notice that (17) is a condition to constrain $\vec{X}_{\mu}$, which is completely independent of $\hat{A}_{\mu}$. If so, (2) should also be interpreted as a condition on $\mathscr{X}_{\mu}$, a consistency condition to keep the colored gluons massless. This strongly implies that (2) can not be used to determine the Abelian potential $\mathscr{A}_{\mu}$. This is a critical defect of the Abelian projection based on the maximal Abelian gauge.

Another disadvantage of (2) is that this tells nothing about the monopole. Obviously we need the monopole potential to test the monopole dominance, but (2) does not provide any information on the monopole. In comparison (10) determines not only the Abelian potential $\hat{A}_{\mu}$ but also the monopole potential $\vec{C}_{\mu}$ uniquely and gauge independently.

An important advantage of (3) is that it can actually "Abelianize" QCD gauge independently [2, 3]. To see this we replace the valence potential $\vec{X}_{\mu}$ by the complex vector field $X_{\mu}$,

$$
\vec{X}_{\mu}=X_{\mu}^{1} \hat{n}_{1}+X_{\mu}^{2} \hat{n}_{2}, \quad X_{\mu}=\frac{1}{\sqrt{2}}\left(X_{\mu}^{1}+i X_{\mu}^{2}\right),
$$

and express the Lagrangian (14) by

$$
\begin{gathered}
\mathcal{L}=-\frac{1}{4} G_{\mu \nu}^{2}-\frac{1}{2}\left|\hat{D}_{\mu} X_{\nu}-\hat{D}_{\nu} X_{\mu}\right|^{2}+i g G_{\mu \nu} X_{\mu}^{*} X_{\nu} \\
-\frac{1}{2} g^{2}\left[\left(X_{\mu}^{*} X_{\mu}\right)^{2}-\left(X_{\mu}^{*}\right)^{2}\left(X_{\nu}\right)^{2}\right] \\
=-\frac{1}{4}\left(G_{\mu \nu}+X_{\mu \nu}\right)^{2}-\frac{1}{2}\left|\hat{D}_{\mu} X_{\nu}-\hat{D}_{\nu} X_{\mu}\right|^{2} \\
B_{\mu}=A_{\mu}+\tilde{C}_{\mu} \\
G_{\mu \nu}=\partial_{\mu} B_{\nu}-\partial_{\nu} B_{\mu}=F_{\mu \nu}+H_{\mu \nu}, \\
\hat{D}_{\mu} X_{\nu}=\left(\partial_{\mu}+i g B_{\mu}\right) X_{\nu} \\
X_{\mu \nu}=-i g\left(X_{\mu}^{*} X_{\nu}-X_{\nu}^{*} X_{\mu}\right) .
\end{gathered}
$$

Clearly this looks like an Abelian gauge theory coupled to the charged vector field $X_{\mu}$, except that this has two Abelian potentials. So the theory becomes a dual gauge theory whose Abelian gauge group is given by $U(1)_{e} \otimes U(1)_{m}$ [2, 3]. Moreover, in this Abelianization the topological structure of the non-Abelian gauge theory is retained in the dual structure, in the magnetic potential $\tilde{C}_{\mu}$.

Actually this Abelianized QCD is not Abelian, because (19) has the full non-Abelian gauge symmetry. To see this let

$$
\begin{gathered}
\vec{\alpha}=\alpha_{1} \hat{n}_{1}+\alpha_{2} \hat{n}_{2}+\theta \hat{n}, \quad \alpha=\frac{1}{\sqrt{2}}\left(\alpha_{1}+i \alpha_{2}\right), \\
\vec{C}_{\mu}=-\frac{1}{g} \hat{n} \times \partial_{\mu} \hat{n}=-C_{\mu}^{1} \hat{n}_{1}-C_{\mu}^{2} \hat{n}_{2}, \\
C_{\mu}=\frac{1}{\sqrt{2}}\left(C_{\mu}^{1}+i C_{\mu}^{2}\right) .
\end{gathered}
$$

Then (19) is invariant not only under the active gauge transformation described by

$$
\begin{gathered}
\delta A_{\mu}=\frac{1}{g} \partial_{\mu} \theta-i\left(C_{\mu}^{*} \alpha-C_{\mu} \alpha^{*}\right), \quad \delta \tilde{C}_{\mu}=-\delta A_{\mu}, \\
\delta X_{\mu}=0,
\end{gathered}
$$

but also under the following passive gauge transformation described by

$$
\begin{gathered}
\delta A_{\mu}=\frac{1}{g} \partial_{\mu} \theta-i\left(X_{\mu}^{*} \alpha-X_{\mu} \alpha^{*}\right), \quad \delta \tilde{C}_{\mu}=0, \\
\delta X_{\mu}=\frac{1}{g} \hat{D}_{\mu} \alpha-i \theta X_{\mu} .
\end{gathered}
$$

This tells that the Abelianized QCD not only retains the full non-Abelian gauge symmetry, but also has an enlarged (both active and passive) gauge symmetries.

Notice that in the conventional (Savvidy's) Abelianization based on the decomposition (11), $\mathscr{A}_{\mu}$ and $\mathscr{X}_{\mu}$ transform (not to $B_{\mu}$ and $X_{\mu}$ but) to $A_{\mu}$ and $X_{\mu}^{\prime}=$ $\left(A_{\mu}^{1}+i A_{\mu}^{2}\right) / \sqrt{2}$. So QCD becomes an Abelian gauge theory made of $A_{\mu}$ (without $\tilde{C}_{\mu}$ ) which is coupled to $X_{\mu}^{\prime}$. Adopting this view Savvidy integrated out $X_{\mu}^{\prime}$ to obtain the QCD effective action, treating $A_{\mu}$ and $X_{\mu}^{\prime}$ as the classical (slow) and quantum (fast) fields.

This view, however, has critical defects. First of all, the separation of $\vec{A}_{\mu}$ to $\mathscr{A}_{\mu}$ and $\mathscr{X}_{\mu}$ is ad hoc, it is not gauge independent. Moreover $\mathscr{A}_{\mu}$ does not have the full gauge degrees of freedom, nor does $\mathscr{X}_{\mu}$ transform covariantly. So the decomposition (1) has no objective meaning.

Worse, the Abelian part here becomes exactly the Maxwell-type U(1) gauge theory. This trivializes QCD to QED which has the massless colored gluons as its source, which fails to take into account the dual structure of the Abelian sub-dynamics of QCD. In particular, this completely neglects the role of non-Abelian monopole, an essential feature of QCD. Clearly this is not QCD. 


\section{SAVVIDY BACKGROUND VERSUS MONOPOLE BACKGROUND}

An important lesson from the above discussion is that there are actually two sources of magnetic background in QCD, the Maxwell-type $F_{\mu \nu}$ and the Dirac-type $H_{\mu \nu}$, because the restricted potential has a dual structure. So we have to specify which background we must choose and explain why we must do so when we calculate the effective action. The old calculations based on (11) completely missed this point because they had only $F_{\mu \nu}$.

To understand why this is so important, consider the gauge transformation which inverts the color direction which we call the color reflection,

$$
\left(\hat{n}_{1}, \hat{n}_{2}, \hat{n}_{3}\right) \rightarrow\left(\hat{n}_{1},-\hat{n}_{2},-\hat{n}\right) .
$$

Under this we have

$$
\begin{gathered}
\hat{A}_{\mu}=\mathscr{A}_{\mu}+\vec{C}_{\mu} \rightarrow \hat{A}_{\mu}^{(c)}=-\mathscr{A}_{\mu}+\vec{C}_{\mu}, \\
\vec{X}_{\mu}=X_{\mu}^{1} \hat{n}_{1}+X_{\mu}^{2} \hat{n}_{2} \rightarrow \vec{X}_{\mu}^{(c)}=X_{\mu}^{1} \hat{n}_{1}-X_{\mu}^{2} \hat{n}_{2} .
\end{gathered}
$$

Notice that the electric and magnetic parts in $\hat{A}_{\mu}$ transform oppositely.

In the Abelianized QCD this translates to

$$
\begin{gathered}
A_{\mu} \rightarrow A_{\mu}, \quad \tilde{C}_{\mu} \rightarrow-\tilde{C}_{\mu}, \\
X_{\mu}=\frac{1}{\sqrt{2}}\left(X_{\mu}^{1}+i X_{\mu}^{2}\right) \rightarrow X_{\mu}^{*}=\frac{1}{\sqrt{2}}\left(X_{\mu}^{1}-i X_{\mu}^{2}\right), \\
D_{\mu} X_{\mu}=\left[\partial_{\mu}+i g\left(A_{\mu}+\tilde{C}_{\mu}\right)\right] X_{\mu} \\
\rightarrow\left(D_{\mu} X_{\mu}\right)^{*}=\left[\partial_{\mu}+i g\left(-A_{\mu}+\tilde{C}_{\mu}\right)\right] X_{\mu}^{*} .
\end{gathered}
$$

Clearly this tells that the red gluon $X_{\mu}$ changes to the blue gluon $X_{\mu}^{*}$ (and vise versa), and the monopole becomes anti-monopole, under the color reflection. As importantly the gauge interaction of $A_{\mu}$ changes the signature but that of $\tilde{C}_{\mu}$ remains invariant. Furthermore, $F_{\mu \nu}$ and $H_{\mu \nu}$ transform oppositely under the color reflection.

Obviously this color reflection which we call "the Cparity" is exactly the color charge conjugation which changes the color of the valence gluons. But what is important about this C-parity is that it must be a symmetry of QCD, because (23) is a gauge transformation. In particular, this C-parity guarantees the non-Abelian gauge invariance in the Abelianized QCD [2, 3].

Now, since the Maxwell potential in QED has negative charge conjugation, $A_{\mu}$ must have negative C-parity. This means that $A_{\mu}$ and $\tilde{C}_{\mu}$ (and $F_{\mu \nu}$ and $H_{\mu \nu}$ ) in (19) have negative and positive C-parity, respectively. This immediately tells that the magnetic background made of $F_{\mu \nu}$ is not gauge invariant. This, of course, was the problem of the SNO vacuum [14].

As importantly this assures that the monopole background made of $H_{\mu \nu}$ is gauge invariant, because it has positive C-parity. So we do not have to construct "the Copenhagen vacuum" to obtain a gauge invariant background [15]. QCD already has a natural candidate of gauge invariant background, the monopole background. And this is what we need for the dual Meissner effect.

The two potentials $A_{\mu}$ and $\tilde{C}_{\mu}$ have another important difference. Consider the space inversion (the parity P) $\vec{x} \rightarrow-\vec{x}$. Under this $A_{\mu}$ behaves as an ordinary vector, so that it has negative parity. But in spherical coordinates this space inversion changes $(r, \theta, \phi)$ to $(r, \pi-\theta, \pi+\phi)$, so that we must have

$$
\begin{gathered}
\hat{n}_{1}=\cos \phi \hat{\theta}-\sin \phi \hat{\phi} \rightarrow \hat{n}_{1}^{(p)}=-\cos \phi \hat{\theta}-\sin \phi \hat{\phi}, \\
\hat{n}_{2}=\sin \phi \hat{\theta}+\cos \phi \hat{\phi} \rightarrow \hat{n}_{2}^{(p)}=-\sin \phi \hat{\theta}+\cos \phi \hat{\phi}, \\
\tilde{C}_{\mu}=-\frac{1}{g} \hat{n}_{1} \cdot \partial_{\mu} \hat{n}_{2}=-\frac{1}{g}(1-\cos \theta) \partial_{\mu} \phi \\
\rightarrow \tilde{C}_{\mu}^{(p)}=-\frac{1}{g}(1+\cos \theta) \partial_{\mu} \phi .
\end{gathered}
$$

This shows that the monopole becomes an anti-monopole under the space inversion. This might be somewhat unexpected. But this is because $\hat{r}$ changes to $-\hat{r}$ under the space inversion, so that the monopole charge $\pi_{2}\left(S^{2}\right)$ changes the signature.

To understand the physical meaning of this, notice that the monopole potential $\vec{C}_{\mu}$ in (4) is not sensitive to the signature of $\hat{n}$. This means that in QCD there is no distinction between monopole and anti-monopole. In fact the monopole charge in QCD is determined only up to the Weyl reflection (i.e., the color reflection), so that monopole and anti-monopole are gauge equivalent to each other [28]. So the space inversion does not change the physical content of $\tilde{C}_{\mu}$, which means that it should be interpreted as an axial vector which has positive parity.

From this we conclude that $J^{P C}$ of the electric potential $A_{\mu}$ becomes $1^{--}$while $J^{P C}$ of the magnetic potential $\tilde{C}_{\mu}$ becomes $1^{++}$. This tells that the background made of the electric potential violates not only the gauge invariance but also the parity, which is a conserved quantum number in strong interaction. In contrast, the background made of the magnetic potential is gauge invariant and parity conserving.

Before we close this section we make a few remarks. First, the monopole background should really be interpreted as the monopole-antimonople background, since monopole and anti-monopole are gauge equivalent in QCD. This must be contrasted with Dirac's theory of monopole, where monopole and anti-monopole are clearly different.

Second, the Savvidy background violates the parity (as well as the gauge invariance). This is another critical defect, because the Savvidy vacuum would violate the parity which we know is a good quantum number of strong interaction. As far as we understand this defect has never been pointed out before. 
Third, our anaysis tells that physically the space inversion is equivalent to the isospace inversion. There is always a residual $\mathrm{U}(1)$ gauge transformation which connects two inversions. This kind of space-isospace locking (e.g., the spin-isospin locking) of course is common in monopole and skyrmion physics [29].

\section{SNO EFFECTIVE ACTION: A REVIEW}

To understand what is wrong with the old calculations of the QCD effective action it is important to know how they calculated it. So in this section we briefly review the old calculations. To obtain the effective action Savvidy and others have divided the classical and quantum parts by (10), and integrated out the quantum part in the presence of the Savvidy background [13, 14, 16, 17]

$$
\hat{F}_{\mu \nu}^{(b)}=\bar{F}_{\mu \nu} \hat{n}_{0}, \quad \bar{F}_{\mu \nu}=H \delta_{[\mu}^{1} \delta_{\nu]}^{2},
$$

where $\hat{n}_{0}=(0,0,1)$ is a constant Abelian direction and $H$ is a constant chromomagnetic field in $z$-direction.

In this case the calculation of the functional determinant of the gluon loop integral amounts to finding the energy spectrum of a charged vector field moving around a constant magnetic field. It is well-known that this energy spectrum is given by [30]

$$
\mathcal{E}=2 g H\left(n+\frac{1}{2}-q S_{3}\right)+k^{2},
$$

where $S_{3}$ and $k$ are the spin and momentum of the vector fields in the direction of the magnetic field, and $q= \pm 1$ is the charge (positive and negative) of the vector fields. This is schematically shown in Fig. 2. Notice that for both charges the energy spectrum contains negative eigenvalues (tachyonic modes) which violate the causality.

From (28) we obtain the integral expression of the effective action

$$
\begin{aligned}
& \Delta \mathcal{L}=\lim _{\epsilon \rightarrow 0} \frac{\mu^{2}}{16 \pi^{2}} \int_{0}^{\infty} \frac{d t}{t^{2-\epsilon}} \frac{g H}{\sinh \left(g H t / \mu^{2}\right)} \\
& \times\left[\exp \left(-2 g H t / \mu^{2}\right)+\exp \left(+2 g H t / \mu^{2}\right)\right],
\end{aligned}
$$

where $\mu^{2}$ is a dimensional parameter. Notice that the second term has a severe infra-red divergence, which we can regularize with the standard $\zeta$-function regularization. From the generalized $\zeta$-function [31]

$$
\zeta(s, \lambda)=\sum_{n=0}^{\infty} \frac{1}{(n+\lambda)^{s}}=\frac{1}{\Gamma(s)} \int_{0}^{\infty} \frac{x^{s-1} \exp (-\lambda x)}{1-\exp (-x)} d x
$$

we have

$$
\Delta \mathcal{L}=\lim _{\epsilon \rightarrow 0} \frac{\mu^{2}}{8 \pi^{2}} g H \int_{0}^{\infty} \frac{d t}{t^{2-\epsilon}}
$$

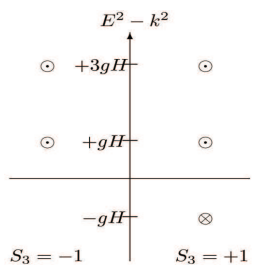

(A)

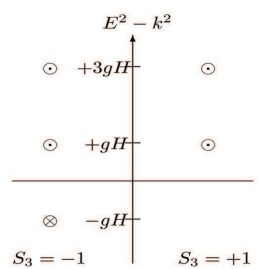

(B)
FIG. 2: The energy eigenvalues of charged vector fields in a constant magnetic field. (A) and (B) show the eigenvalues of positively and negatively charged $(q= \pm 1)$ vector field. In both cases the eigenvalues contain tachyonic modes when $k^{2}<g H$.

$$
\begin{aligned}
& \times \frac{\exp \left(-3 g H t / \mu^{2}\right)+\exp \left(+g H t / \mu^{2}\right)}{1-\exp \left(-2 g H t / \mu^{2}\right)} \\
&= \lim _{\epsilon \rightarrow 0} \frac{(g H)^{2}}{4 \pi^{2}}\left(\frac{2 g H}{\mu^{2}}\right)^{-\epsilon} \Gamma(\epsilon-1) \\
& \times\left[\zeta\left(\epsilon-1, \frac{3}{2}\right)+\zeta\left(\epsilon-1,-\frac{1}{2}\right)\right] \\
&=\frac{11 g^{2} H^{2}}{48 \pi^{2}}\left(\frac{1}{\epsilon}-\gamma+1-\ln \frac{2 g H}{\mu^{2}}\right) \\
&-\frac{g^{2} H^{2}}{4 \pi^{2}}\left(2 \zeta^{\prime}\left(-1, \frac{3}{2}\right)-i \frac{\pi}{2}\right),
\end{aligned}
$$

where $\zeta^{\prime}=\frac{d \zeta}{d s}(s, \lambda)$. With this we arrive at the SNO effective action [13, 14, 16]

$$
\begin{gathered}
\mathcal{L}_{e f f}=-\frac{H^{2}}{2}-\frac{11 g^{2} H^{2}}{48 \pi^{2}}\left(\ln \frac{g H}{\mu^{2}}-c\right)+i \frac{g^{2} H^{2}}{8 \pi}, \\
c=1-\ln 2-\frac{24}{11} \zeta^{\prime}\left(-1, \frac{3}{2}\right)=0.94556 \ldots,
\end{gathered}
$$

which contains the well-known imaginary part which destablizes the SNO vacuum [14]. Obviously the imaginary part originates from the tachyonic eigenstates.

There have been many attempts to restore the stability to the SNO vacuum [15-17]. Actually we can remove the imaginary part calculating the effective action using different methods, for example, with the infra-red regularization by causality [32, 33]. But the difficult part is to explain exactly what is wrong with the conventional (Savvidy's) calculations and the $\zeta$-function regularization.

Of course, this instability is not the only problem of the SNO vacuum. A more serious problem is that it is not gauge invariant, because it is not invariant under the color reflection. Furthermore, it violates the parity, as we have pointed out. So the SNO vacuum can not be identified as the $\mathrm{QCD}$ vacuum, even if it can be made stable.

Because of this Nielsen and Olesen have introduced "the Copenhagen vacuum", a randomly oriented blockwise Savvidy background, and conjectured that such background could provide a gauge invariant (and thus 
hopefully stable) QCD vacuum [15]. Unfortunately the Copenhagen vacuum is not gauge invariant either, because we can not restore the gauge invariance by violating it randomly blockwise.

\section{GAUGE INVARIANT CALCULATION OF QCD EFFECTIVE ACTION}

The above discussion tells that the old calculations of QCD effective action which renders the SNO vacuum have many defects. For example, the decomposition of the classical and quantum parts (1) is not gauge independent. But this is not a serious defect, because in practical calculations one may always choose a convenient gauge.

There are two critical defects in the old calculations. First, the Savvidy background is neither gauge invariant nor parity conserving, as we have emphasized. The gauge invariant and parity conserving background is the monopole background, but the old calculations based on (11) could not choose this background because it has no place for the monopole.

Second, the gauge invariance is completely overlooked in the calculation of the gluon functional determinant. In particular, the color reflection invariance (the C-parity) is not correctly implemented in the old calculations. And the instability of the SNO vacuum originates from this mistake.

To show how to calculate the effective action correctly, we adopt the gauge independent Abelian decomposition (10) and integrate out the colored gluons in the presence of the gauge invariant monopole background. The advantage of this, of course, is that the whole process of the calculation becomes explicitly gauge independent.

To integrate out $\vec{X}_{\mu}$ we have to fix the (quantum) gauge, and we choose the generalized Lorentz gauge

$$
\vec{F}=\hat{D}_{\mu} \vec{X}_{\mu}=0, \quad \mathcal{L}_{g f}=-\frac{1}{2 \xi}\left(\hat{D}_{\mu} \vec{X}_{\mu}\right)^{2}
$$

The corresponding Faddeev-Popov determinant can be expressed by

$$
\begin{gathered}
M_{a b}^{F P}=\frac{\delta F_{a}}{\delta \alpha^{b}} \simeq\left(\hat{D}_{\mu} D_{\mu}\right)_{a b}-\hat{n}_{a}\left(\hat{n} \cdot \hat{D}_{\mu} D_{\mu}\right)_{b} \\
=\Pi_{a c}\left(\hat{D}_{\mu} D_{\mu}\right)_{c b}, \quad \Pi_{a b}=\delta_{a b}-\hat{n}_{a} \hat{n}_{b} .
\end{gathered}
$$

Notice that $\Pi_{a b}$ is the projection operator which projects out the $\hat{n}$ component. With this we have

$$
\begin{gathered}
\exp \left[i S_{\text {eff }}\left(\hat{A}_{\mu}\right)\right]=\int \mathcal{D} \vec{X}_{\mu} \mathcal{D} \vec{c} \mathcal{D} \vec{c}^{*} \\
\exp \left\{i \int \left[-\frac{1}{4} \hat{F}_{\mu \nu}^{2}-\frac{1}{4}\left(\hat{D}_{\mu} \vec{X}_{\nu}-\hat{D}_{\nu} \vec{X}_{\mu}\right)^{2}\right.\right. \\
-\frac{g}{2} \hat{F}_{\mu \nu} \cdot\left(\vec{X}_{\mu} \times \vec{X}_{\nu}\right)-\frac{g^{2}}{4}\left(\vec{X}_{\mu} \times \vec{X}_{\nu}\right)^{2}
\end{gathered}
$$

$$
\left.\left.+\vec{c}^{*} \hat{D}_{\mu} D_{\mu} \vec{c}-\frac{1}{2 \xi}\left(\hat{D}_{\mu} \vec{X}_{\mu}\right)^{2}\right] d^{4} x\right\}
$$

where $\vec{c}$ and $\vec{c}^{*}$ are the ghost fields which are orthogonal to $\hat{n}$. Here we need only the ghosts which are orthogonal to $\hat{n}$ because they come from the gauge fixing of the valence gluons which are orthogonal to $\hat{n}$.

Obviously the effective action (34) becomes gauge invariant under the transformation (12), if the ghost fields transform as

$$
\delta \vec{c}=-\alpha \times \vec{c}, \quad \delta \vec{c}^{*}=-\alpha \times \vec{c}^{*} .
$$

So the whole process of the calculation becomes gauge independent.

To demonstrate the monopole condensation, of course, we have to choose the gauge invariant and parity conserving monopole background,

$$
\hat{F}_{\mu \nu}^{(b)}=\bar{H}_{\mu \nu} \hat{n}, \quad \bar{H}_{\mu \nu}=H \delta_{[m}^{1} \delta_{n]}^{2} .
$$

This should be compared with the Savvidy background (27) which is not gauge invariant nor parity conserving. To be general, however, we will let $\bar{H}_{\mu \nu}$ arbitrary but constant in the following.

With this the gluon and ghost loop integrals give the following deteminants (at one-loop level)

$$
\begin{gathered}
\operatorname{Det}^{-\frac{1}{2}} K_{\mu \nu, a b}=\operatorname{Det}^{-\frac{1}{2}}\left(-g_{\mu \nu} \bar{D}_{a b}^{2}-2 g \epsilon_{a b c} \bar{H}_{\mu \nu} \hat{n}^{c}\right), \\
\operatorname{Det} M_{a b}=\operatorname{Det}\left(-\bar{D}_{a b}^{2}\right),
\end{gathered}
$$

where $\bar{D}_{\mu}$ is the covariant derivative defined by the monopole background. From this we have

$$
\Delta S=\frac{i}{2} \ln \operatorname{Det} K-i \ln \operatorname{Det} M
$$

So the calculation of the determinants becomes a crucial part to obtain the effective action.

To calculate the functional determinants, notice that

$$
\begin{gathered}
\ln \operatorname{Det} K=\operatorname{Tr} \ln \left(-g_{\mu \nu} \bar{D}_{a b}^{2}\right) \\
+\operatorname{Tr} \ln \left[g_{\mu \nu} \delta_{a b}+2 g \bar{H}_{\mu \nu}\left(\frac{N}{\bar{D}^{2}}\right)_{a b}\right]=4 \operatorname{Tr} \ln \left(-\bar{D}_{a b}^{2}\right) \\
+\operatorname{Tr} \sum_{n=1}^{\infty} \frac{(-1)^{n+1}}{n}\left[\left(2 g \bar{H}_{\mu \nu} \frac{N}{\bar{D}^{2}}\right)^{n}\right]_{a b} \\
N_{a b}=\epsilon_{a b c} n_{c} .
\end{gathered}
$$

We can simplify this to

$$
\begin{gathered}
\ln \operatorname{Det} K=4 \operatorname{Tr} \ln \left(-\bar{D}_{a b}^{2}\right) \\
+\operatorname{Tr} \ln \left[\delta_{a b}+4 a^{2}\left(\frac{N}{\bar{D}^{2}}\right)_{a b}^{2}\right]+\operatorname{Tr} \ln \left[\delta_{a b}-4 b^{2}\left(\frac{N}{\bar{D}^{2}}\right)_{a b}^{2}\right] \\
=\ln \operatorname{Det}\left[\left(-\bar{D}^{2}+2 i a N\right)\left(-\bar{D}^{2}-2 i a N\right)\right]_{a b}
\end{gathered}
$$




$$
\begin{gathered}
+\ln \operatorname{Det}\left[\left(-\bar{D}^{2}+2 b N\right)\left(-\bar{D}^{2}-2 b N\right)\right]_{a b}, \\
a=\frac{g}{2} \sqrt{\sqrt{\bar{H}^{4}+(\bar{H} \tilde{\bar{H}})^{2}}+\bar{H}^{2}}, \\
b=\frac{g}{2} \sqrt{\sqrt{\bar{H}^{4}+(\bar{H} \tilde{\bar{H}})^{2}}-\bar{H}^{2} .}
\end{gathered}
$$

Here $\left(-\bar{D}^{2}+2 i a N\right)$ and $\left(-\bar{D}^{2}-2 i a N\right)$ (also $\left(-\bar{D}^{2}+2 b N\right)$ and $\left.\left(-\bar{D}^{2}-2 b N\right)\right)$ correspond to two spin polarization degrees of valence gluons $\left(S_{3}= \pm 1\right)$.

Notice that $a$ and $b$ represent purely magnetic and purely electric background of $\bar{H}_{\mu \nu}$, so that from now on we call them magnetic and electric background, respectively. This should not be confused with the decomposition of $\hat{F}_{\mu \nu}$ to the electric $\left(F_{\mu \nu}\right)$ and magnetic $\left(H_{\mu \nu}\right)$ part.

To proceed, we have to calculate two determinants

$$
\left(-\bar{D}^{2} \pm 2 i a N\right), \quad\left(-\bar{D}^{2} \pm 2 b N\right) .
$$

Now, from

$$
\left(-\bar{D}^{2} \pm 2 i a N\right) \hat{n}=0, \quad\left(-\bar{D}^{2} \pm 2 b N\right) \hat{n}=0,
$$

we may assume that the eigenfunction of the determinants are orthogonal to $\hat{n}$,

$$
\vec{\phi}=\phi_{1} \hat{n}_{1}+\phi_{2} \hat{n}_{2}
$$

Furthermore, with

$$
\begin{gathered}
\bar{D}_{\mu} \hat{n}_{1}=g \bar{C}_{\mu} \hat{n}_{2}, \quad \bar{D}_{\mu} \hat{n}_{2}=-g \bar{C}_{\mu} \hat{n}_{1}, \\
\bar{C}_{\mu}=\frac{1}{2} \bar{H}_{\mu \nu} x^{\nu},
\end{gathered}
$$

we can express the eigenvalue equation of the first determinant by

$$
\begin{gathered}
\left(\begin{array}{c}
\partial_{\mu}^{2}-g^{2} \bar{C}_{\mu}^{2},-g\left(\partial_{\mu} \bar{C}_{\mu}+2 \bar{C}_{\mu} \partial_{\mu}\right) \pm 2 i a \\
g\left(\partial_{\mu} \bar{C}_{\mu}+2 \bar{C}_{\mu} \partial_{\mu}\right) \mp 2 i a, \partial_{\mu}^{2}-g^{2} \bar{C}_{\mu}^{2}
\end{array}\right)\left(\begin{array}{c}
\phi_{1} \\
\phi_{2}
\end{array}\right) \\
=\lambda\left(\begin{array}{c}
\phi_{1} \\
\phi_{2}
\end{array}\right)
\end{gathered}
$$

which can be diagonalized to the Abelian form

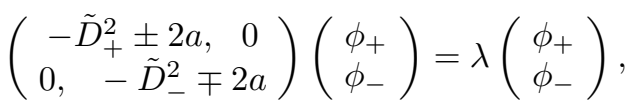

$$
\begin{aligned}
& \phi_{ \pm}=\frac{\phi_{1} \pm i \phi_{2}}{\sqrt{2}}, \quad \tilde{D}_{ \pm}^{2}=\left(\partial_{\mu} \pm i g \bar{C}_{\mu}\right)^{2} .
\end{aligned}
$$

Notice that $\phi_{+}$and $\phi_{-}$are conjugate to each other under the color reflection (23).

Similarly, we can write the eigenvalue equation of the second determinant as

$$
\left(\begin{array}{cc}
-\tilde{D}_{+}^{2} \mp 2 i b, & 0 \\
0, & -\tilde{D}_{-}^{2} \pm 2 i b
\end{array}\right)\left(\begin{array}{c}
\phi_{+} \\
\phi_{-}
\end{array}\right)=\lambda\left(\begin{array}{c}
\phi_{+} \\
\phi_{-}
\end{array}\right) .
$$

From this we have

$$
\begin{gathered}
\operatorname{Det}\left(-\bar{D}^{2} \pm 2 i a N\right)_{a b} \\
=\operatorname{Det}\left(-\tilde{D}_{+}^{2} \pm 2 a\right)\left(-\tilde{D}_{-}^{2} \mp 2 a\right), \\
\operatorname{Det}\left(-\bar{D}^{2} \pm 2 b N\right)_{a b} \\
=\operatorname{Det}\left(-\tilde{D}_{+}^{2} \mp 2 i b\right)\left(-\tilde{D}_{-}^{2} \pm 2 i b\right) .
\end{gathered}
$$

Again, notice that $\left(-\tilde{D}_{+}^{2} \pm 2 a\right) \phi_{+}$and $\left(-\tilde{D}_{+}^{2} \mp 2 i b\right) \phi_{+}$are conjugate to $\left(-\tilde{D}_{-}^{2} \mp 2 a\right) \phi_{-}$and $\left(-\tilde{D}_{-}^{2} \pm 2 i b\right) \phi_{-}$under the color reflection (23). This means that they are gauge equivalent to each other, so that we must have

$$
\begin{aligned}
& \operatorname{Det}\left(-\tilde{D}_{+}^{2} \pm 2 a\right)=\operatorname{Det}\left(-\tilde{D}_{-}^{2} \mp 2 a\right), \\
& \operatorname{Det}\left(-\tilde{D}_{+}^{2} \mp 2 i b\right)=\operatorname{Det}\left(-\tilde{D}_{-}^{2} \pm 2 i b\right) .
\end{aligned}
$$

From this we have

$$
\begin{gathered}
\ln \operatorname{Det} K=2 \ln \operatorname{Det}\left(-\tilde{D}^{2}+2 a\right)\left(-\tilde{D}^{2}-2 a\right) \\
+2 \ln \operatorname{Det}\left(-\tilde{D}^{2}-2 i b\right)\left(-\tilde{D}^{2}+2 i b\right), \\
\ln \operatorname{Det} M=2 \ln \operatorname{Det}\left(-\tilde{D}^{2}\right),
\end{gathered}
$$

where $\tilde{D}_{\mu}=\partial_{\mu}+i g \bar{C}_{\mu}$. Notice that the determinants are expressed entirely by Abelian determinants, with Abelian covariant derivative.

Now, (50) reproduces the well known expression of QCD effective action [16, 17]

$$
\begin{gathered}
\Delta S=i \ln \operatorname{Det}\left(-\tilde{D}^{2}+2 a\right)\left(-\tilde{D}^{2}-2 a\right) \\
+i \ln \operatorname{Det}\left(-\tilde{D}^{2}-2 i b\right)\left(-\tilde{D}^{2}+2 i b\right) \\
-2 i \ln \operatorname{Det}\left(-\tilde{D}^{2}\right),
\end{gathered}
$$

and

$$
\begin{aligned}
\Delta \mathcal{L}= & \lim _{\epsilon \rightarrow 0} \frac{1}{16 \pi^{2}} \int_{0}^{\infty} \frac{d t}{t^{3-\epsilon}} \frac{a b t^{2}}{\sinh \left(a t / \mu^{2}\right) \sin \left(b t / \mu^{2}\right)} \\
& \times\left[\exp \left(-2 a t / \mu^{2}\right)+\exp \left(+2 a t / \mu^{2}\right)\right. \\
+ & \left.\exp \left(+2 i b t / \mu^{2}\right)+\exp \left(-2 i b t / \mu^{2}\right)-2\right] .
\end{aligned}
$$

Notice that the first four terms are the gluon loop contribution, but the last term comes from the ghost loop. When $a=g H$ and $b=0$, this becomes identical to (29). And here again, the second and fourth terms have a severe infra-red divergence.

We emphasize, however, this derivation of (51) has a critical defect. To see this remember that the gluon loop we are supposed to integrate out must be, not the red and blue gluon loops, but the physical gluon loop made of the gauge invariant combination of red and blue gluons. In other words the gluon loop integral $\mathcal{D} \vec{X}_{\mu}$ in (34) should really be understood as the gauge invariant integral $\left(\mathcal{D} \vec{X}_{\mu}\right)_{i}$. But this important point has not been correctly taken into account in (51).

To understand this in detail, consider the case $a=g H$ and $b=0$ shown in Fig. 3. Clearly the color reflection 


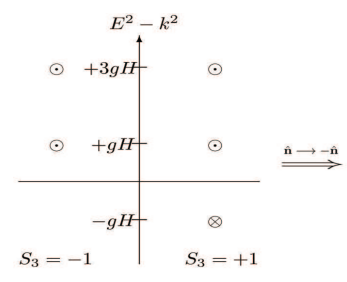

(A)

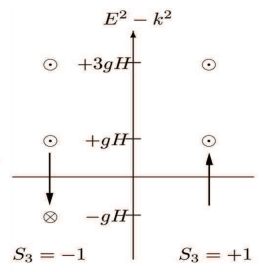

(B)
FIG. 3: The gauge invariant eigenvalues of the gluon functional determinant. (A) and (B) are the C-parity partners, so that they are gauge equivalent for each spin polarization separately. This shows that the negative eigenvalues in both (A) and (B) are not gauge invariant.

(the C-parity) changes (A) to (B), so that they are gauge equivalent. But since this C-parity does not change the spin of the valence gluons, we must require the physical eigenvalues to be invariant under the C-parity for each spin polarization separately,

$$
\begin{aligned}
& \operatorname{Det}\left(-\tilde{D}_{+}^{2}+2 a\right)=\operatorname{Det}\left(-\tilde{D}_{-}^{2}-2 a\right), \\
& \operatorname{Det}\left(-\tilde{D}_{+}^{2}-2 a\right)=\operatorname{Det}\left(-\tilde{D}_{-}^{2}+2 a\right) .
\end{aligned}
$$

Now, obviously the negative eigenvalues for both $S_{3}=$ +1 in (A) and $S_{3}=-1$ in (B) do not satisfy this requirement, so that they must be discarded. This is the C-projection which removes the tachyonic states and restores the gauge invariance of the gluon loop integral. This neglect of gauge invariance is the critical mistake of the conventional calculations $13-16]$.

Notice that the C-parity here plays exactly the same role as the G-parity in string theory. It is well known that the GSO projection (the G-projection) restores the supersymmetry and modular invariance in NSR string by projecting out the tachyonic vacuum [18, 19]. Just like the G-projection in string, the C-projection in $Q C D$ removes the tachyonic modes and restores the gauge invariance of the effective action.

Exactly the same argument applies to $\operatorname{Det}\left(-\tilde{D}_{+}^{2} \mp 2 i b\right)$ and $\operatorname{Det}\left(-\tilde{D}_{-}^{2} \pm 2 i b\right)$. Here again they are the C-parity counterpart of each other, so that we must require

$$
\begin{aligned}
& \operatorname{Det}\left(-\tilde{D}_{+}^{2}-2 i b\right)=\operatorname{Det}\left(-\tilde{D}_{-}^{2}+2 i b\right), \\
& \operatorname{Det}\left(-\tilde{D}_{+}^{2}+2 i b\right)=\operatorname{Det}\left(-\tilde{D}_{-}^{2}-2 i b\right),
\end{aligned}
$$

for each spin polarization separately.

This tells that (51) is incorrect. The correct functional determinant is given by the C-projection of it,

$$
\begin{aligned}
\ln \operatorname{Det} K & =\ln \operatorname{Det}\left[\left(-\hat{D}^{2}+2 i a N\right)\left(-\hat{D}^{2}-2 i a N\right)\right]_{a b} \\
+ & \ln \operatorname{Det}\left[\left(-\hat{D}^{2}+2 b N\right)\left(-\hat{D}^{2}-2 b N\right)\right]_{a b} \\
& =2 \ln \operatorname{Det}\left(-\tilde{D}^{2}+2 a\right)\left(-\tilde{D}^{2}+2 a\right) \\
& +2 \ln \operatorname{Det}\left(-\tilde{D}^{2}-2 i b\right)\left(-\tilde{D}^{2}-2 i b\right)
\end{aligned}
$$

This leads us to

$$
\begin{gathered}
\Delta S=i \ln \operatorname{Det}\left[\left(-\tilde{D}^{2}+2 a\right)\left(-\tilde{D}^{2}+2 a\right)\right] \\
+i \ln \operatorname{Det}\left[\left(-\tilde{D}^{2}-2 i b\right)\left(-\tilde{D}^{2}-2 i b\right)\right] \\
-2 i \ln \operatorname{Det}\left(-\tilde{D}^{2}\right)
\end{gathered}
$$

and

$$
\begin{aligned}
\Delta \mathcal{L} & =\lim _{\epsilon \rightarrow 0} \frac{1}{8 \pi^{2}} \int_{0}^{\infty} \frac{d t}{t^{3-\epsilon}} \frac{a b t^{2} / \mu^{4}}{\sinh \left(a t / \mu^{2}\right) \sin \left(b t / \mu^{2}\right)} \\
& \times\left[\exp \left(-2 a t / \mu^{2}\right)+\exp \left(+2 i b t / \mu^{2}\right)-1\right] .
\end{aligned}
$$

This is the new integral expression of QCD effective action, which should be compared with (52). Obviously the C-projection makes (57) gauge invariant. As importantly, it removes the infra-red divergence of (52).

At the first glance this might be surprising, but actually is not so. The gauge invariance implies the confinement which implies the generation of a mass parameter, which should make the theory infra-red finite. So it is natural that the gauge invariance makes (57) infra-red finite.

When $a=0$ or $b=0$, the integration is straightforward. For pure magnetic background we have

$$
\Delta \mathcal{L}=\lim _{\epsilon \rightarrow 0} \frac{a / \mu^{2}}{8 \pi^{2}} \int_{0}^{\infty} \frac{d t}{t^{2-\epsilon}} \frac{\exp \left(-2 a t / \mu^{2}\right)}{\sinh \left(a t / \mu^{2}\right)},
$$

so that 32,33

$$
\mathcal{L}_{\text {eff }}=-\frac{a^{2}}{2 g^{2}}-\frac{11 a^{2}}{48 \pi^{2}}\left(\ln \frac{a}{\mu^{2}}-c\right) .
$$

This is identical to the SNO effective action (31), except that it no longer has the imaginary part. This, of course, assures the stability of the monopole condensation.

For the pure electric background we have

$$
\Delta \mathcal{L}=\lim _{\epsilon \rightarrow 0} \frac{b / \mu^{2}}{8 \pi^{2}} \int_{0}^{\infty} \frac{d t}{t^{2-\epsilon}} \frac{\exp \left(+2 i b t / \mu^{2}\right)}{\sin \left(b t / \mu^{2}\right)},
$$

so that [32, 33]

$$
\mathcal{L}_{e f f}=\frac{b^{2}}{2 g^{2}}+\frac{11 b^{2}}{48 \pi^{2}}\left(\ln \frac{b}{\mu^{2}}-c\right)-i \frac{11 b^{2}}{96 \pi} .
$$

To summarize we have

$$
\mathcal{L}_{\text {eff }}= \begin{cases}-\frac{a^{2}}{2 g^{2}}-\frac{11 a^{2}}{48 \pi^{2}}\left(\ln \frac{a}{\mu^{2}}-c\right), & b=0 \\ \frac{b^{2}}{2 g^{2}}+\frac{11 b^{2}}{48 \pi^{2}}\left(\ln \frac{b}{\mu^{2}}-c\right) & \\ -i \frac{11 b^{2}}{96 \pi}, & a=0\end{cases}
$$

Notice that when $a=0$ the imaginary part has a negative signature, which implies the pair annihilation of 
gluons. This must be contrasted with the QED effective action where the electron loop integral generates a positive imaginary part [34, 35]. The positive imaginary part in QED means the pair creation which generates the screening. On the other hand in QCD we must have the anti-screening to explain the asymptotic freedom, and the negative imaginary part is what we need [32, 33].

Observe that (59) and (61) are related by the electricmagnetic duality [32]. We can obtain one from the other simply by replacing $a$ with $-i b$ and $b$ with $i a$. This duality, which states that the effective action should be invariant under the replacement

$$
a \rightarrow-i b, \quad b \rightarrow i a,
$$

was first discovered in the QED effective action [35]. But subsequently this duality has also been shown to exist in the QCD effective action 32]. This tells that the duality should be regarded as a fundamental symmetry of the effective action of gauge theory, Abelian and non-Abelian. The importance of this duality is that it provides a very useful tool to check the self-consistency of the effective action. The fact that (59) and (61) are related by the duality assures that they are self-consistent.

Clearly we can arrive at the same conclusion using the Abelianized QCD Lagrangian (19). The only difference is that in the Abelianized QCD, we integrate out the complex valence gluons 32, 33].

The evaluation of the integral (57) for arbitrary $a$ and $b$ is not easy. Even in the "simpler" QED, the integration of the effective action has been completed only recently [35]. But we can do the integration for arbitrary $a b \neq 0$, and prove that the monopole condensation is the true minimum of the effective potential [36].

\section{INFRA-RED REGULARIZATION BY CAUSALITY}

The above analysis tells that the gauge invariance (the C-projection) resolves the instability of the SNO vacuum. But notice that this instability is not just the problem of the SNO vacuum but the problem of the QCD effective action, which originates from the infra-red instability of QCD itself.

To amplify this notice that the infra-red divergence in (29) was obtained with the Savvidy background, but the one in (52) was obtained with the monopole background. This tells that the infra-red divergence could be viewed as a generic feature of QCD effective action which arises in both Savvidy and monopole background, whose origin can be traced back to the absence of a mass scale. If so, one may ask if there is any way to control this infra-red instability.

Of course we have already pointed out that this is due to the mishandling of the gluon functional determinant.
Indeed the C-projection removes this infra-red divergence by changing (52) to (57). But we may still ask if there is another way to remove the infra-red instability directly from (52). The answer is yes [32, 33].

To see this remember that the effective action can be viewed as the generating functional of the vacuum to vacuum transition amplitude in the presence of the monopole background $\vec{C}_{\mu}$ [27],

$$
\begin{aligned}
& \exp \left[i S_{e f f}\right]=\left.\left\langle\Omega_{+} \mid \Omega_{-}\right\rangle\right|_{\vec{C}_{\mu}} \\
& =\left.\sum_{\left|n_{i}\right\rangle}\left\langle\Omega_{+} \mid n_{i}\right\rangle\left\langle n_{i} \mid \Omega_{-}\right\rangle\right|_{\vec{C}_{\mu}},
\end{aligned}
$$

where $\left|\Omega_{ \pm}\right\rangle$is the vacuum at $t= \pm \infty,\left|n_{i}\right\rangle$ is a complete orthonomal set of colored gluon states, and the gluon loop integral in (52) corresponds to the summation. To calculate this amplitude, of course, we must use physical $\left|\Omega_{ \pm}\right\rangle$and physical $\left|n_{i}\right\rangle$. But obviously the tachyonic states are not physical because they violate the causality, so that they should be excluded from the summation. The question is how. We can do that performing the integral correctly, with the infra-red regularization by causality [32].

To do that we start from the integral (52), and consider the pure magnetic background first. To impose the infra-red regularization by causality, we go to the Minkowski-time with the Wick rotation, and find [32]

$$
\begin{gathered}
\Delta \mathcal{L}=\Delta \mathcal{L}_{+}+\Delta \mathcal{L}_{-}, \\
\Delta \mathcal{L}_{+}=-\lim _{\epsilon \rightarrow 0} \frac{a / \mu^{2}}{16 \pi^{2}} \int_{0}^{\infty} \frac{d t}{t^{2-\epsilon}} \frac{\exp \left(-2 i a t / \mu^{2}\right)}{\sin \left(a t / \mu^{2}\right)} \\
\Delta \mathcal{L}_{-}=-\lim _{\epsilon \rightarrow 0} \frac{a / \mu^{2}}{16 \pi^{2}} \int_{0}^{\infty} \frac{d t}{t^{2-\epsilon}} \frac{\exp \left(+2 i a t / \mu^{2}\right)}{\sin \left(a t / \mu^{2}\right)}
\end{gathered}
$$

In this form the infra-red divergence has disappeared, but now we face an ambiguity in choosing the correct contours of the integrals.

Fortunately we can resolve this ambiguity imposing the causality. To see this notice that the two integrals $\Delta \mathcal{L}_{+}$and $\Delta \mathcal{L}_{-}$originate from two determinants $\left(-\tilde{D}^{2}+\right.$ $2 a)$ and $\left(-\tilde{D}^{2}-2 a\right)$, but the standard causality argument (with the familiar Feynman prescription $p^{2} \rightarrow p^{2}-i \epsilon$ ) requires us to identify $2 a$ in the first determinant as $2 a-i \epsilon$ but in the second determinant as $2 a+i \epsilon$. This tells that the poles in the first integral in (65) should lie above the real axis, while the poles in the second integral should lie below the real axis. From this we conclude that the two integrals become complex conjugate to each other. This guarantees that $\Delta \mathcal{L}$ is explicitly real, without any imaginary part. Moreover the real part of $\Delta \mathcal{L}_{+}$and $\Delta \mathcal{L}_{-}$ must be the same. So with this infra-red regularization by causality we obtain [32]

$$
\mathcal{L}_{\text {eff }}=-\frac{a^{2}}{2 g^{2}}-\frac{11 a^{2}}{48 \pi^{2}}\left(\ln \frac{a}{\mu^{2}}-c\right),
$$


which is identical to (59).

For the pure electric background the infra-red regularization by causality, with a similar reasoning, yields [32, 33.

$$
\mathcal{L}_{e f f}=\frac{b^{2}}{2 g^{2}}+\frac{11 b^{2}}{48 \pi^{2}}\left(\ln \frac{b}{\mu^{2}}-c\right)-i \frac{11 b^{2}}{96 \pi},
$$

which is identical to (61). This confirms that we can indeed control the infra-red instability of the effective action by causality. It is really remarkable that two completely independent principles, the gauge invariance and the causality, produce exactly the same result.

Of course, this infra-red regularization by causality could make the SNO vaccum stable. But this is not the point. As we have emphasized, the SNO vacuum can not be the QCD vacuum independent of whether it is stable or not. It violates the gauge invariance and the parity. Nor does it tell that the $\zeta$-function regularization is wrong. It works well with (57). This merely tells that there is a physical regularization which can correct the infra-red instability of (52), independent of what is the QCD vacuum.

\section{PERTURBATIVE CONFIRMATION}

There is another powerful way to calculate the imaginary part in the effective action. This is because in massless gauge theories (in particular in QCD) the imaginary part of the one-loop effective action is of the order of $g^{2}$, so that we can actually calculate it perturbatively [32, 37]. Considering that the effective action is essentially non-perturbative, this is unexpected. But this has been demonstrated in both massless QED and QCD [32, 33].

In QED Schwinger has obtained the following effective action perturbatively to the order $e^{2}[34]$

$$
\begin{gathered}
\triangle S_{Q E D}=\frac{e^{2}}{16 \pi^{2}} \int d^{4} p F_{\mu \nu}(p) F_{\mu \nu}(-p) \\
\times \int_{0}^{1} d v \frac{v^{2}\left(1-v^{2} / 3\right)}{\left(1-v^{2}\right)+4 m^{2} / p^{2}},
\end{gathered}
$$

where $m$ is the electron mass. From this he observed that when $-p^{2}>4 m^{2}$ the integrand develops a pole at $v^{2}=1+4 m^{2} / p^{2}$ which generates an imaginary part, and explained how to calculate it. But notice that in the massless limit, the pole moves to $v=1$. In this case the pole contribution to the imaginary part is reduced by a half, and we obtain

$$
\left.\operatorname{Im} \mathcal{L}_{Q E D}\right|_{m=0}= \begin{cases}0 & b=0 \\ \frac{b^{2}}{48 \pi} & a=0\end{cases}
$$

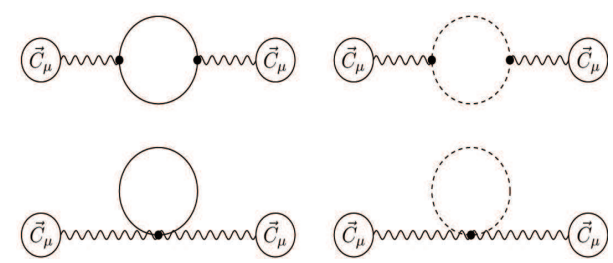

FIG. 4: The Feynman diagrams that contribute to the effective action at $g^{2}$ order. Here the straight line and the dotted line represent the valence gluon and the ghost, respectively.

This is exactly what we obtain from the non-perturbative QED effective action in the massless limit [32, 33].

In QCD we can find the imaginary part either by calculating the one-loop Feynman diagrams directly, or by evaluating the integral (52) to the order $g^{2}$ using Schwinger's method. Here we do this with the Feynman diagrams.

For an arbitrary monopole background there are four Feynman diagrams that contribute to the order $g^{2}$ which are shown in Fig. 4. The sum of these diagrams (in the Feynman gauge with dimensional regularization) gives us [27]

$$
\begin{aligned}
\Delta S=- & \frac{11 g^{2}}{96 \pi^{2}} \int d^{4} p H_{\mu \nu}(p) H_{\mu \nu}(-p) \\
& \times\left[\ln \left(\frac{p^{2}}{\mu^{2}}\right)+C_{1}\right],
\end{aligned}
$$

where $C_{1}$ is a regularization-dependent constant. Clearly the imaginary part could only arise from the logarithmic term $\ln \left(p^{2} / \mu^{2}\right)$, so that for a space-like $p^{2}$ (with $\left.\mu^{2}>0\right)$ the effective action has no imaginary part. But a space-like $p^{2}$ corresponds to a magnetic background. This means that (52) has no imaginary part when $b=0$, at least at the order $g^{2}$.

To evaluate the imaginary part for an electric background we have to make the analytic continuation of (68) to a time-like $p^{2}$. In this case the causality (again with the Feynman prescription $p^{2} \rightarrow p^{2}-i \epsilon$ ) dictates us to have

$$
\begin{aligned}
& \ln \left(\frac{p^{2}}{\mu^{2}}\right) \rightarrow \lim _{\epsilon \rightarrow 0} \ln \left(\frac{p^{2}-i \epsilon}{\mu^{2}}\right) \\
& =\ln \left(\frac{\left|p^{2}\right|}{\mu^{2}}\right)-i \frac{\pi}{2} \quad\left(p^{2}<0\right),
\end{aligned}
$$

so that we obtain

$$
\text { Im } \Delta \mathcal{L}=\left\{\begin{array}{cc}
0 & (b=0), \\
-\frac{11 b^{2}}{96 \pi} & (a=0) .
\end{array}\right.
$$

Obviously this is identical to (62). And we can reproduce the same result using the Schwinger's method [33].

It might look surprising that both the infra-red regularization by causality and the perturbative calculation 
produce the same imaginary part. But this is natural, because both are based on the causality.

This confirms that we can control the infra-red instability of QCD effective action if we treat it correctly. In particular this tells that the tachyonic modes in (52) are indeed unphysical mirage which should not have been there in the first place. They come into the calculation of the functional determinant by default.

\section{DIMENSIONAL TRANSMUTATION}

The effective action (59) generates the much desired dimensional transmutation in QCD, the phenomenon Coleman and Weinberg first observed in massless scalar QED [12, 27]. To demonstrate this notice that the effective action (59) provides the following effective potential

$$
V=\frac{a^{2}}{2 g^{2}}\left[1+\frac{11 g^{2}}{24 \pi^{2}}\left(\ln \frac{a}{\mu^{2}}-c\right)\right] .
$$

So, defining the running coupling $\bar{g}$ by 32,33$]$

$$
\left.\frac{\partial^{2} V}{\partial a^{2}}\right|_{a=\bar{\mu}^{2}}=\frac{1}{\bar{g}^{2}}
$$

we have

$$
\begin{gathered}
\frac{1}{\bar{g}^{2}}=\frac{1}{g^{2}}+\frac{11}{24 \pi^{2}}\left(\ln \frac{\bar{\mu}^{2}}{\mu^{2}}-c+\frac{3}{2}\right), \\
\beta(\bar{\mu})=\bar{\mu} \frac{\partial \bar{g}}{\partial \bar{\mu}}=-\frac{11 \bar{g}^{3}}{24 \pi^{2}} .
\end{gathered}
$$

This is exactly the same $\beta$-function in perturbative $\mathrm{SU}(2)$ QCD which assures the asymptotic freedom [38].

In terms of the running coupling the renormalized potential is given by

$$
V_{\text {ren }}=\frac{a^{2}}{2 \bar{g}^{2}}\left[1+\frac{11 \bar{g}^{2}}{24 \pi^{2}}\left(\ln \frac{a}{\bar{\mu}^{2}}-\frac{3}{2}\right)\right],
$$

which generates a non-trivial local minimum at

$$
\langle a\rangle=\bar{\mu}^{2} \exp \left(-\frac{24 \pi^{2}}{11 \bar{g}^{2}}+1\right) .
$$

Notice that with $\bar{\alpha}_{s}=1$ we have

$$
\frac{\langle a\rangle}{\bar{\mu}^{2}}=0.48988 \ldots
$$

This is nothing but the dimensional transmutation by the monopole condensation. The corresponding effective potential is plotted in Fig. 5, where we have assumed $\bar{\alpha}_{s}=1$ and $\bar{\mu}^{2}=1$.

Nelsen and Olesen have suggested that the existence of the unstable tachyonic modes are closely related with the asymptotic freedom in QCD [14]. Our analysis tells that this is not true. Obviously the asymptotic freedom shown in (73) follows from the stable monopole condensation in the absence of tachyons.

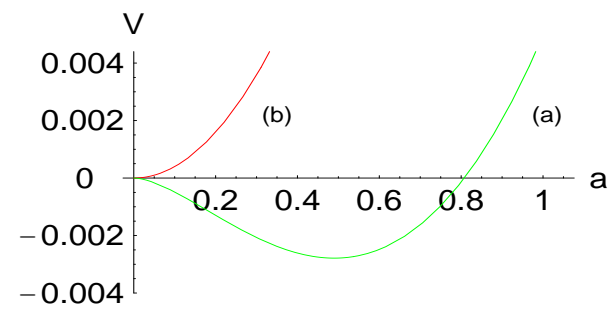

FIG. 5: The effective potential of SU(2) QCD in the monopole background. Here (a) is the effective potential and (b) is the classical potential.

\section{DISCUSSION}

Establishing the dimensional transmutation by the monopole condensation in QCD has been extremely difficult to attain. Savvidy and many others had the correct idea, but made serious mistakes in implementing the idea. Their calculations (in particular their Abelian decomposition) were not gauge independent. This in itself may not be a serious problem, but the old calculations have two critical defects. First, they have chosen the wrong background which is neither gauge invariant nor parity conserving. This is the problem of the SNO background. Second, they have failed to impose the gauge invariance in the gluon loop integral. This is the problem of the infra-red instability of the effective action.

The first problem arises because the old calculations neglected the fact that there are actually two possible magnetic backgrounds in QCD, the SNO background and the monopole background, but only the monopole background qualifies to be the QCD vacuum because only this background is gauge invariant and parity conserving. This is because they did not take into account the fact that the restricted potential has a dual structure.

The second problem, the infra-red instability of the effective action, originates from the fact that the old calculations failed to implement the gauge invariance in the calculation of the gluon functional determinant properly. In this paper we have shown how the C-projection makes the functional determinant gauge invariant and removes the tachyonic modes which cause the effective action unstable. This assures the stable monopole condensation.

The Abelian decomposition (10) provides a perfect setting for us to calculate the one-loop effective action of QCD. This decomposition separates not only colored potential but also the monopole gauge independently, and allows us to choose the gauge invariant monopole background. Moreover it also tells exactly how each term transforms under the gauge transformation (the color reflection), and tells how to obtain the gauge invariant effective action with the color reflection. So using this decomposition, we can calculate the correct effective action and establish the stable monopole condensation. 
An unavoidable consequence of the monopole condensation is the existence of two magnetic glueballs, the $0^{++}$ and $1^{++}$modes of the vacuum fluctuation, which are not made of the valence gluons. This is because the monopole condensation induces two scales, the correlation length of the monopoles and the penetration length of the color flux [3]. Obviously they are similar to the Higgs scalar and the massive photon that we have in spontaneous symmetry breaking, and it is very difficult to avoid these modes if we believe in the monopole condensation. The challenge now is to confirm (or disprove) the existence of these magnetic glueballs experimentally.

The existence of the tachyonic modes which caused the infra-red instability of the effective action has been a headache, the Gordian knot, in QCD. It was there, and nobody knew how to resolve this puzzle. In fact it has been thought that this infra-red instability is an essential characteristic, a sacred feature, of QCD which comes from the absence of a mass parameter. But in this paper we have shown that the tachyons are an unphysical mirage which should not have been there in the first place. They disappear when we calculate the effective action correctly. The gauge invariance, the infra-red regularization by causality, and the perturbative calculation of the imaginary part all confirm that there is no infra-red instability in the QCD effective action.

As we have emphasized, in physics tachyons appear when we do something improper or choose something unphysical. In spontaneous symmetry breaking tachyons appear when we choose the false vacuum. Similarly, in NSR string we have the tachyonic vacuum when we do not impose the modular invariance and supersymmetry with the G-projection [18, 19]. Here we have the same situation. The tachyons appear when we we do not implement the gauge invariance to the gluon functional determinant correctly. This tells that there is nothing mysterious about the tachyons in QCD.

It is not surprising that the gauge invariance plays the key role to generate the stable monopole condensation. From the beginning the gauge invariance has been the main motivation for the confinement. It is this gauge invariance which forbids any colored object from the physical spectrum of QCD. So it is only natural that the gauge invariance assures the stability of the monopole condensation, and thus the confinement of color.

The Abelian dominance conjecture has been a very popular confinement mechanism [4, 5]. But actually it is a misleading and confusing conjecture. Obviously it is hollow unless it tells what is the Abelian part, and the maximal Abelian gauge (2) does not tell what is that. The gauge independent Abelian projection (3) does tell what is the Abelian part, but here the Abelian part has a dual structure. So we have to tell which is important in the Abelian dominance.

Our analysis confirms that it is the monopole which provides the confinement. And this has been endorsed by the recent lattice calculation [8, 9]. This is made possible because we have the Abelian projection (3) which projects out (not only the Abelian potential but also) the monopole potential gauge independently.

It must be emphasized, however, that the monopole condensation should really be understood as the monopole-antimonopole condensation. This is because in QCD the monopole and anti-monopole are gauge equivalent, because they are the C-parity partners [28].

One may wonder if there is any classical monopoleantimonopole configuration which can suggest the stable monopole condensation. Indeed there is. It has been shown that a pair of monopole-antimonopole strings becomes stable under the quantum fluctuation, if the distance between two strings becomes less than a critical distance [39]. This strongly implies that monopoleantimonopole pairs can be stable against the quantum fluctuation.

At this point one may ask what is wrong with the SNO effective action. Obviously it is not the effective action of QCD. But we can certainly say that it is the effective action of a U(1) gauge theory coupled to a massless charged vector field which has no charge conjugation (color reflection) invariance. This is not QCD, but actually a sick theory. It is well known that such theory (a theory of massless charged vector field which has no nonAbelian gauge invariance) is ill-defined. So there is nothing wrong with the SNO effective action, except that it is the effective action of a sick theory. And the problems of the SNO vacuum are the symptoms of this sickness.

It is truly remarkable (and surprising) that the principles of quantum field theory allow us to demonstrate confinement within the framework of QCD. There has been a proof of confinement in a supersymmetric QCD [40]. Our analysis shows that we can actually establish the existence of the confinement phase within the conventional QCD, with the existing principles of quantum field theory. This should be interpreted as a most spectacular triumph of quantum field theory itself.

We conclude with the following remarks:

1. To show that the monopole condensation is the true vacuum of $\mathrm{QCD}$, we have to integrate the effective action (57) for an arbitrary chromo-electromagnetic background. This is not easy but doable, and we can show that indeed the monopole background remains a true minimum of the effective potential, at least at one-loop level [36, 41].

2. In this paper we have neglected the quarks. We simply remark that the quarks, just as in the asymptotic freedom, tend to destabilize the monopole condensation. But if the number of quarks are small enough, the condensation remains stable. In fact the stability puts exactly the same constraint on the number of quarks as the asymptotic freedom [36, 41].

3. In real QCD, of course, we have to deal with $\mathrm{SU}(3)$. We can do this, and the generic feature of the $\mathrm{SU}(2)$ 
QCD, in particular the dimensional transmutation by the monopole condensation, remains the same [41].

The calculation of the QCD effective action for $a b \neq 0$ which includes the contribution of the quark loop will be published separately [41].

\section{Acknowledgements}

This work is supported in part by the Basic Science Research Program through the National Research Foundation of Korea funded by the Ministry of Education, Science, and Technology (2012-002-134).
[1] Y. Nambu, Phys. Rev. D10, 4262 (1974); S. Mandelstam, Phys. Rep. 23C, 245 (1976); A. Polyakov, Nucl. Phys. B120, 429 (1977).

[2] Y. M. Cho, Phys. Rev. D21, 1080 (1980). See also Y. S. Duan and M. L. Ge, Sci. Sinica 11,1072 (1979).

[3] Y. M. Cho, Phys. Rev. Lett. 46, 302 (1981); Phys. Rev. D23, 2415 (1981); W. S. Bae, Y. M. Cho, and S. W. Kimm, Phys. Rev. D65, 025005 (2002).

[4] G. 't Hooft, Nucl. Phys. B190, 455 (1981).

[5] A Kronfeld, G. Schierholz, and U. Wiese, Nucl. Phys. B293, 461 (1987); A Kronfeld, M. Laursen, G. Schierholz, and U. Wiese, Phys. Lett. B198, 516 (1987).

[6] T. Suzuki and I. Yotsuyanagi, Phys. Rev. D42, 4257 (1990); G. Bali, V. Bornyakov, M. Mueller-Preussker, and K. Schilling, Phys. Rev. D54, 2863 (1996).

[7] Y. M. Cho, Phys. Rev. D62, 074009 (2000).

[8] S. Kato, K. Kondo, T. Murakami, A. Shibata, T. Shinohara, and S. Ito, Phys. Lett. B632, 326 (2006).

[9] S. Ito, S. Kato, K. Kondo, T. Murakami, A. Shibata, and T. Shinohara, Phys. Lett. B645, 67 (2007); B653, 101 (2007); B669, 107 (2008).

[10] T. DeGrand and D. Toussaint, Phys. Rev. D22, 2478 (1980).

[11] V. Bornyakov, H. Ichie, Y. Mori, D. Pleiter, M. Polikarpov, G. Schierholz, T. Streuer, H. Stuben, and T. Suzuki, Phys. Rev. D70, 054506 (2004); V. Bornyakov, H. Ichie, Y. Koma, Y. Mori, Y. Nakamura, D. Pleiter, M. Polikarpov, G. Schierholz, T. Streuer, H. Stuben, and T. Suzuki, Phys. Rev. D70, 074511 (2004).

[12] S. Coleman and E. Weinberg, Phys. Rev. D7, 1888 (1973).

[13] G. K. Savvidy, Phys. Lett. B71, 133 (1977).

[14] N. Nielsen and P. Olesen, Nucl. Phys. B144, 485 (1978).

[15] N. Nielsen and P. Olesen, Nucl. Phys. B160, 380 (1979); C. Rajiadakos, Phys. Lett. B100, 471 (1981).

[16] W. Dittrich and M. Reuter, Phys. Lett. B128, 321, (1983); B144, 99 (1984); C. Flory, Phys. Rev. D28, 1425 (1983); S. K. Blau, M. Visser, and A. Wipf, Int. J. Mod. Phys. A6, 5409 (1991); M. Reuter, M. G. Schmidt, and C. Schubert, Ann. Phys. 259, 313 (1997).

[17] A. Yildiz and P. Cox, Phys. Rev. D21, 1095 (1980); M. Claudson, A. Yilditz, and P. Cox, Phys. Rev. D22, 2022 (1980); J. Ambjorn and R. Hughes, Phys. Lett. B113, 305 (1982).

[18] F. Gliozzi, J. Scherk, and D. Olive, Nucl. Phys. B122, 253 (1983).

[19] See, e.g., M. Green, J. Schwarz, and E. Witten, Superstring Theory Vol. I, Cambridge University Press, 1987; M. Kaku, Introduction to Superstrings, Springer-Verlag
(New York), 1988.

[20] T. T. Wu and C. N. Yang, Phys. Rev. D12, 3845 (1975).

[21] Y. M. Cho, Phys. Rev. Lett. 44, 1115 (1980).

[22] L. Faddeev and A. Niemi, Phys. Rev. Lett. 82, 1624 (1999); Phys. Lett. B449, 214 (1999).

[23] S. Shabanov, Phys. Lett. B458, 322 (1999); B463, 263 (1999); H. Gies, Phys. Rev. D63, 125023 (2001).

[24] R. Zucchini, Int. J. Geom. Meth. Mod. Phys. 1, 813 (2004).

[25] W. S. Bae, Y. M. Cho, and S. W. Kim, Phys. Rev. D65, 025005 (2001).

[26] B. de Witt, Phys. Rev. 162, 1195 (1967); 1239 (1967).

[27] See for example, C. Itzikson and J. Zuber, Quantum Field Theory (McGraw-Hill) 1985; M. Peskin and D. Schroeder, An Introduction to Quantum Field Theory (Addison-Wesley) 1995; S. Weinberg, Quantum Theory of Fields (Cambridge University Press) 1996.

[28] Y. M. Cho, Phys. Lett. B115, 125 (1982).

[29] A. Goldhaber, Phys. Rev. Lett. 36, 1122 (1976); E. Witten, Nucl. Phys. B223, 422 (1983).

[30] W. Tsai and A. Yildiz, Phys. Rev. D4, 3643 (1971); T Goldman and W. Tsai, ibid, 3648 (1971).

[31] See, for example, I. Gradshteyn and I. Ryzhik, Table of Integrals, Series, and Products, edited by A. Jeffery (Academic Press) 1994; M. Abramowitz and I. Stegun, Handbook of Mathematical Functions, (Dover) 1970.

[32] Y. M. Cho and D. G. Pak, Phys. Rev. D65, 074027 (2002); Y. M. Cho, H. W. Lee, and D. G. Pak, Phys. Lett. B525, 347 (2002).

[33] Y. M. Cho, M. L. Walker, and D. G. Pak, JHEP 05, 073 (2004); Y. M. Cho and M. L. Walker, Mod. Phys. Lett A19, 2707 (2004).

[34] J. Schwinger, Phys. Rev. 82, 664 (1951).

[35] Y. M. Cho and D. G. Pak, Phys. Rev. Lett. 86, 1947 (2001); 91, 039151; W. S. Bae, Y. M. Cho, and D. G. Pak, Phys. Rev. D64, 017303 (2001).

[36] Y. M. Cho and D. G. Pak, hep-th/0006051. See also Y. M. Cho and D. G. Pak, in Proceedings of TMU-Yale Symposium on Dynamics of Gauge Fields, edited by T. Appelquist and H. Minakata (Universal Academy Press, Tokyo) (1999).

[37] V. Schanbacher, Phys. Rev. D26, 489 (1982).

[38] D. Gross and F. Wilczek, Phys. Rev. Lett. 26, 1343 (1973); H. Politzer, Phys. Rev. Lett. 26, 1346 (1973).

[39] Y. M. Cho and D. G. Pak, Phys. Lett. B 525, 347 (2006).

[40] N. Seiberg and E. Witten, Nucl. Phys. B426, 19 (1994); B431, 484 (1994).

[41] Y. M. Cho, D. G. Pak, and Pengming Zhang, to be published. 